\title{
Supplementary Data sets
}

Title: Sporadic inclusion body myositis underling mitochondrial dysfunction ameliorated by mitochondrial homing drug, MA-5

\author{
Yoshitsugu Oikawa ${ }^{1,2}$, Rumiko Izumi ${ }^{3}$, Masashi Koide ${ }^{4}$, Yoshihiro \\ Hagiwara $^{4}$, Makoto Kanzaki ${ }^{5}$, Naoki Suzuki ${ }^{3}$, Koichi Kikuchi ${ }^{2}$, \\ Tetsuro Matsuhashi ${ }^{1}$, Yukako Akiyama ${ }^{2}$, Mariko Ichijo ${ }^{2}$, Takafumi \\ Toyohara ${ }^{2,6}$, Takehiro Suzuki ${ }^{2,6}$, \\ Eikan Mishima ${ }^{2}$, Yasutoshi Akiyama ${ }^{2}$, Yoshiaki Ogata ${ }^{6,7}$, \\ Chitose Suzuki $^{2}$, Hironori Hayashi ${ }^{8,9}$, Eiichi N. Kodama ${ }^{8}$, \\ Ken-ichiro Hayashi ${ }^{10}$, Eiji Itoi ${ }^{4}$, Masashi Aoki ${ }^{3}$, Shigeo Kure ${ }^{1} \&$ \\ Takaaki Abe ${ }^{* 2,6,7}$
}

${ }^{1}$ Department of Pediatrics, Tohoku University Graduate School of Medicine, Sendai, 980-8574, Japan 2Division of Nephrology, Endocrinology and Vascular Medicine, Tohoku University Graduate School of Medicine,

Sendai 980-8574, Japan

${ }^{3}$ Division of Neurology, Tohoku University Graduate School of Medicine, Sendai, 980-8574, Japan

${ }^{4}$ Department of Orthopedics, Tohoku University Graduate School of Medicine, Sendai, 980-8574, Japan

5Department of Biomedical Engineering, Tohoku University Graduate School of Biomedical Engineering,

Sendai, 980-8574, Japan

${ }^{6}$ Department of Medical Science, Tohoku University Graduate School of Biomedical Engineering,

Sendai, 980-8574, Japan

${ }^{7}$ Department of Clinical Biology and Hormonal Regulation, Tohoku University Graduate School of Medicine,

Sendai 980-8574, Japan

${ }^{8}$ Division of Infectious Diseases, International Institute of Disaster Science, Graduate School of Medicine,

Tohoku Medical Megabank Organization, Tohoku University, Sendai 980-8574, Japan

${ }^{9}$ Department of Intelligent Network for Infection Control, Graduate School of Medicine, Tohoku University,

Sendai 980-8574, Japan

${ }^{10}$ Department of Biochemistry, Okayama University of Science, Okayama 700-0005, Japan 


\section{Supplementary Figure 1}

A. Normal myoblast

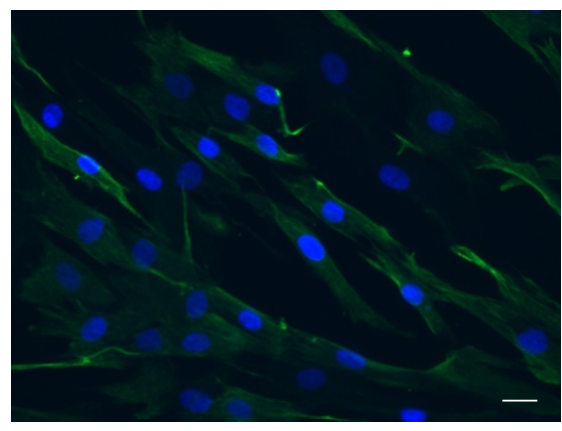

Desmin

B. sIBM1 myoblast

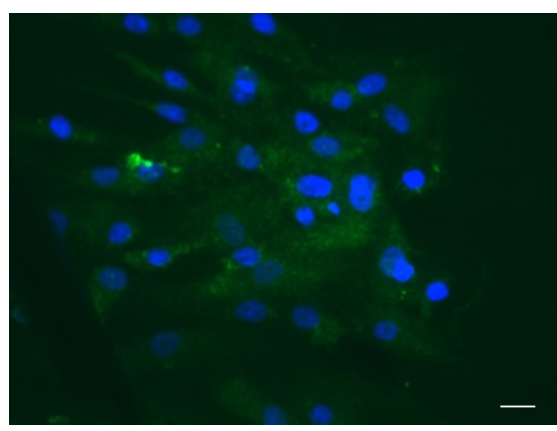

Desmin

C. sIBM2 myoblast

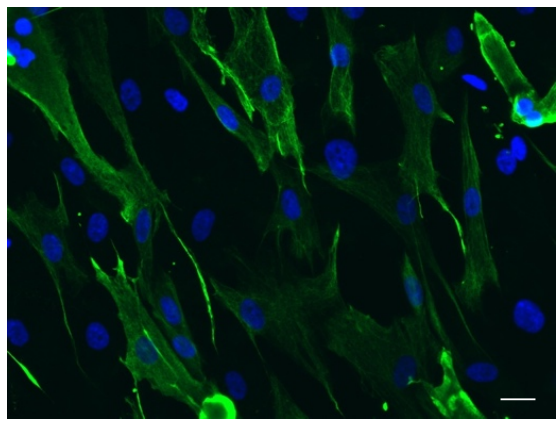

Desmin

D. sIBM3 myoblast

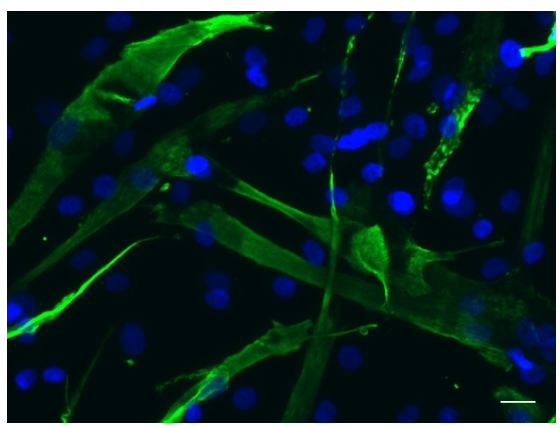

Desmin

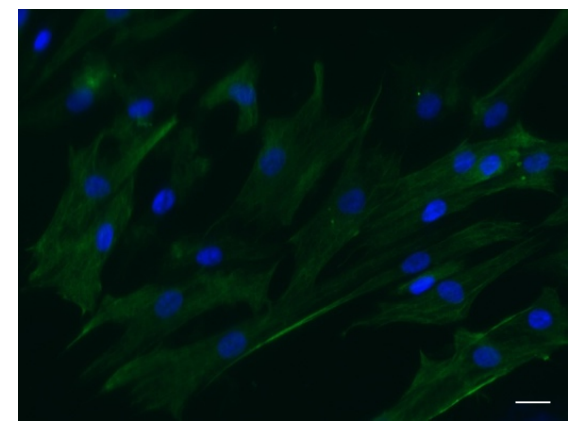

MF20

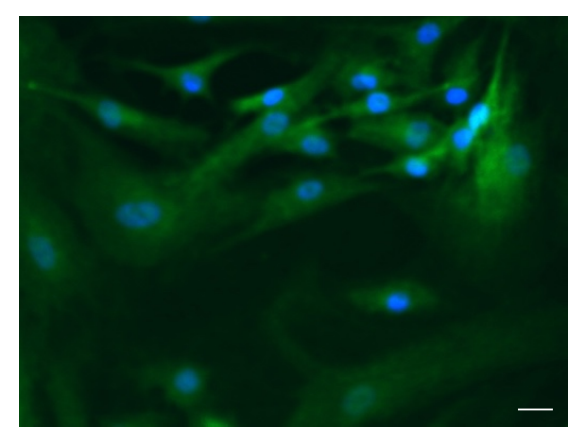

MF20

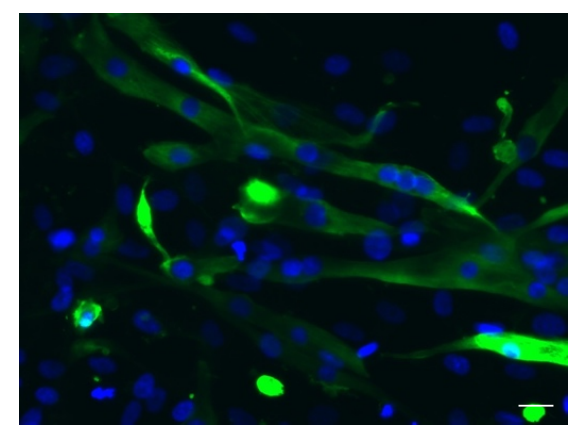

MF20

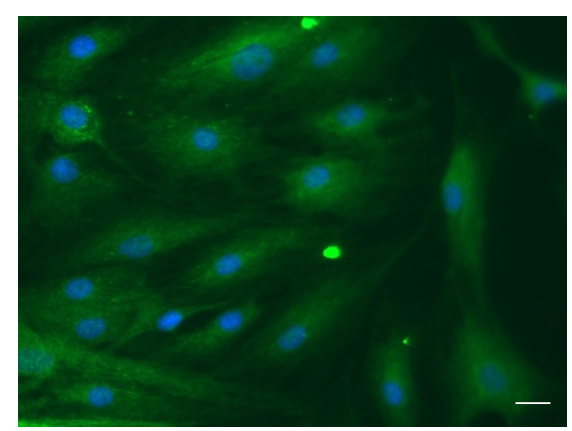

MF20

\section{Figure S1}

Immunostaining in sIBM patient's myoblasts.

Scale bars represent $50 \mu \mathrm{m}$. 


\section{Supplementary Figure 2}

\section{Case2}
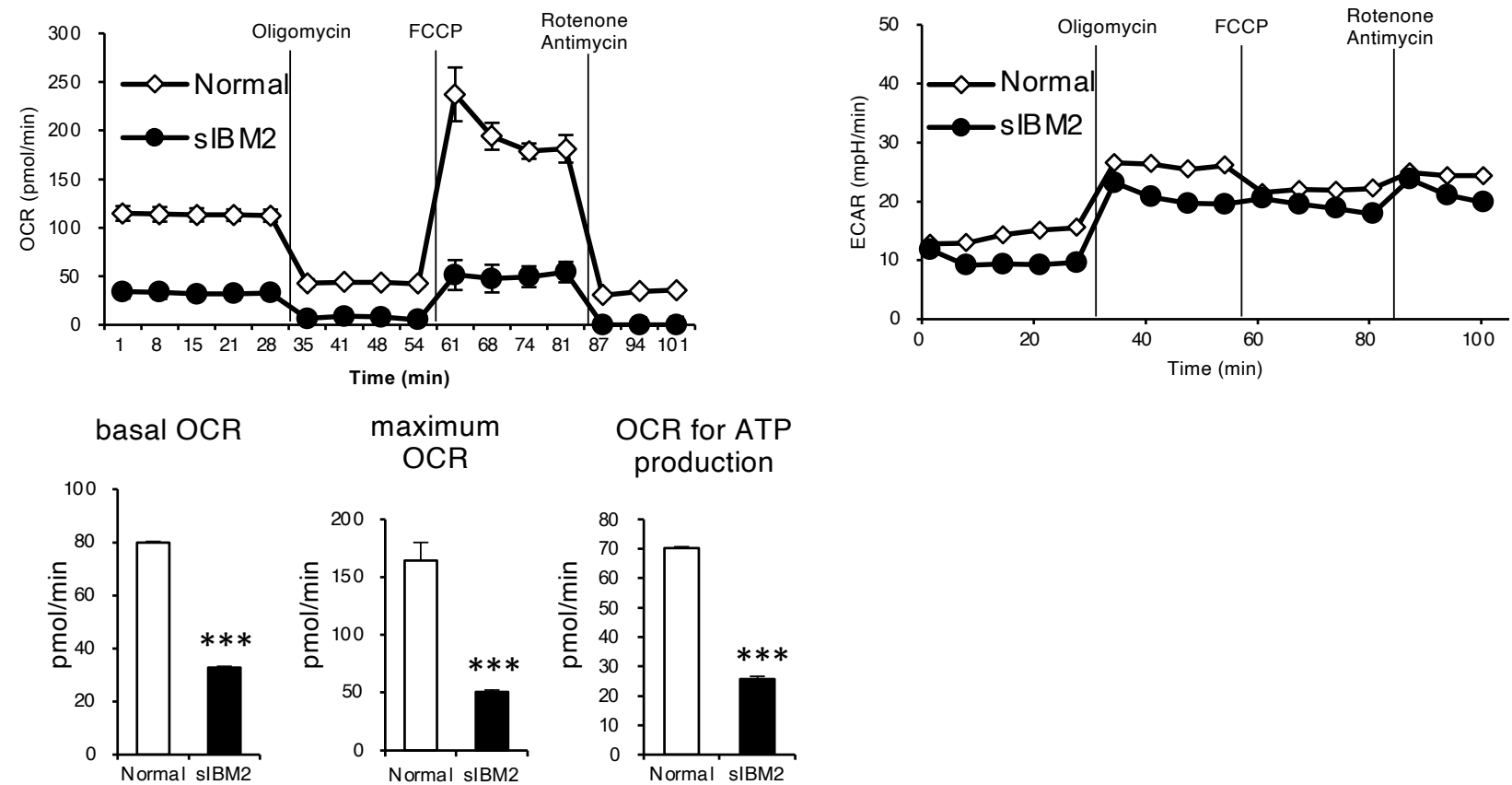

\section{Case3}
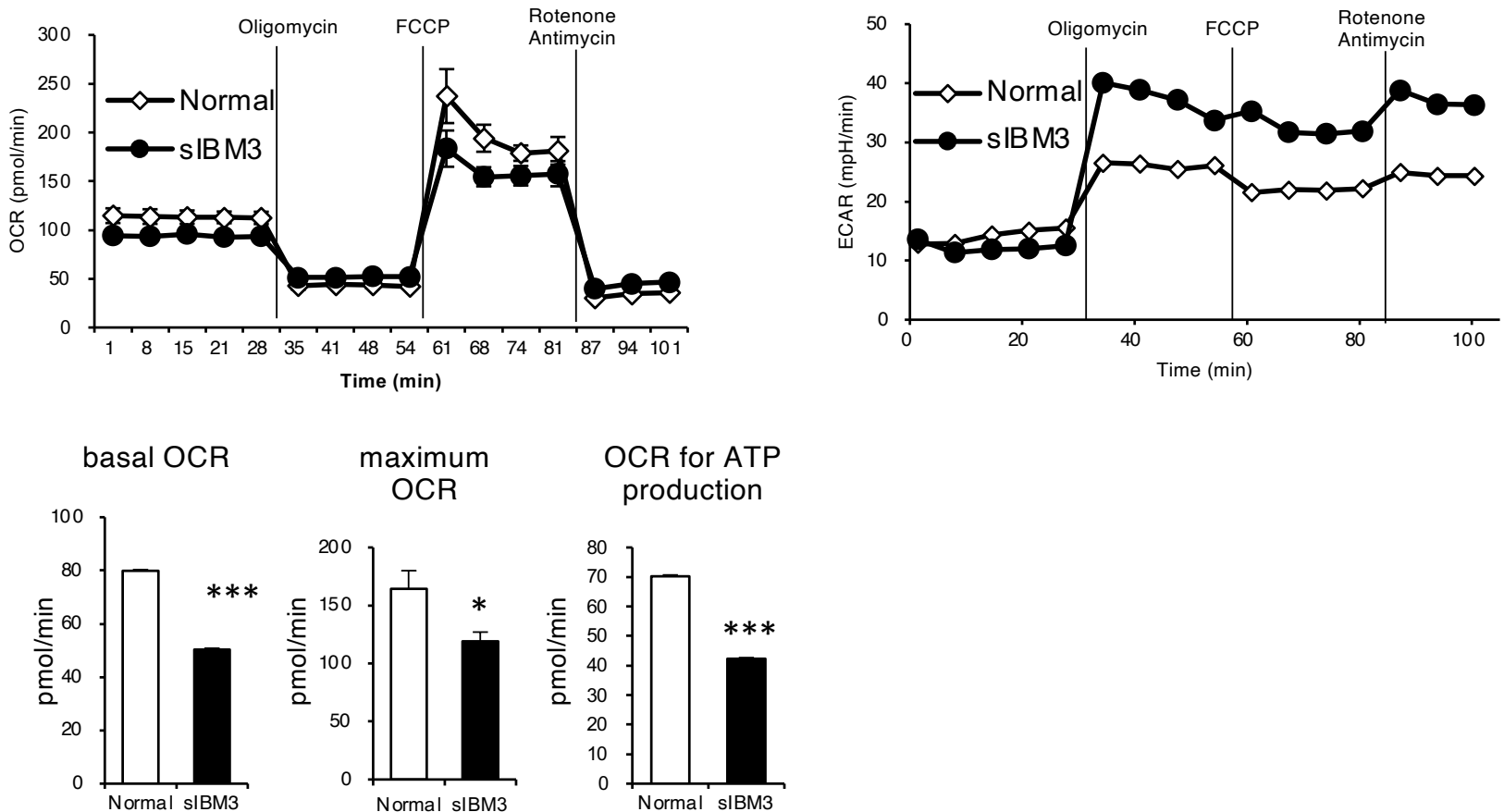

\section{Figure S2}

sIBM myoblasts on mitochondrial respiration.

Bioenergetic assay with normal and sIBM patient myoblasts (sIBM2, sIBM3) in comparison with OCR (left) and ECAR (right). The data represent the mean $\pm \mathrm{SEM}$. ${ }^{*} p<0.05$, *** $p<$ 0.001 (unpaired two-tailed Student's $t$-test versus Normal). 


\section{Supplementary Figure 3}

Case 2
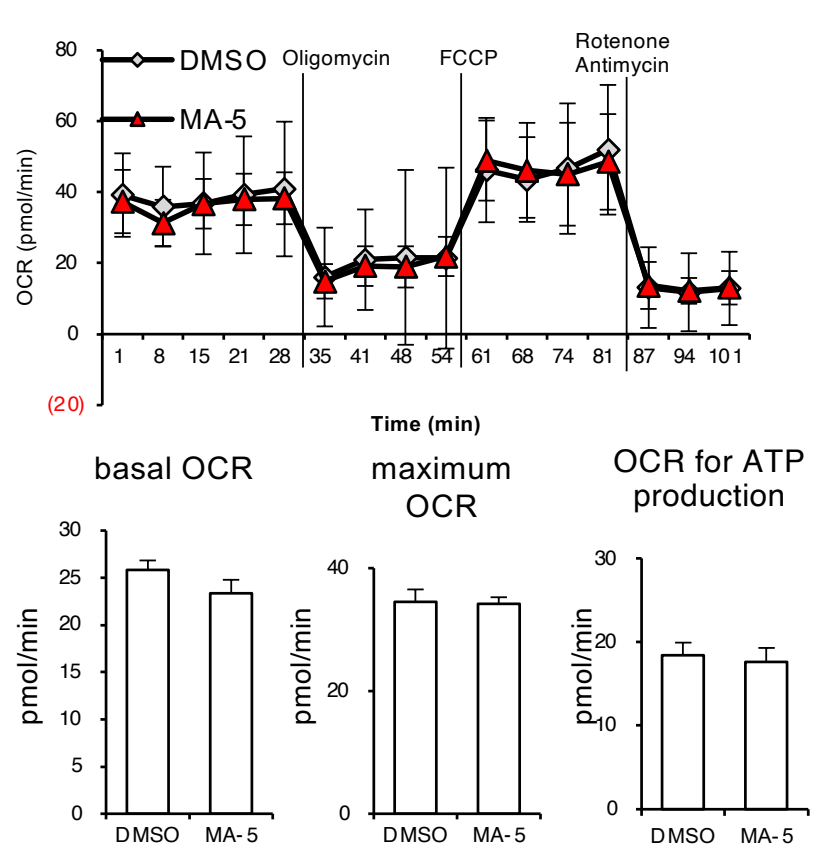

\section{Case 3}
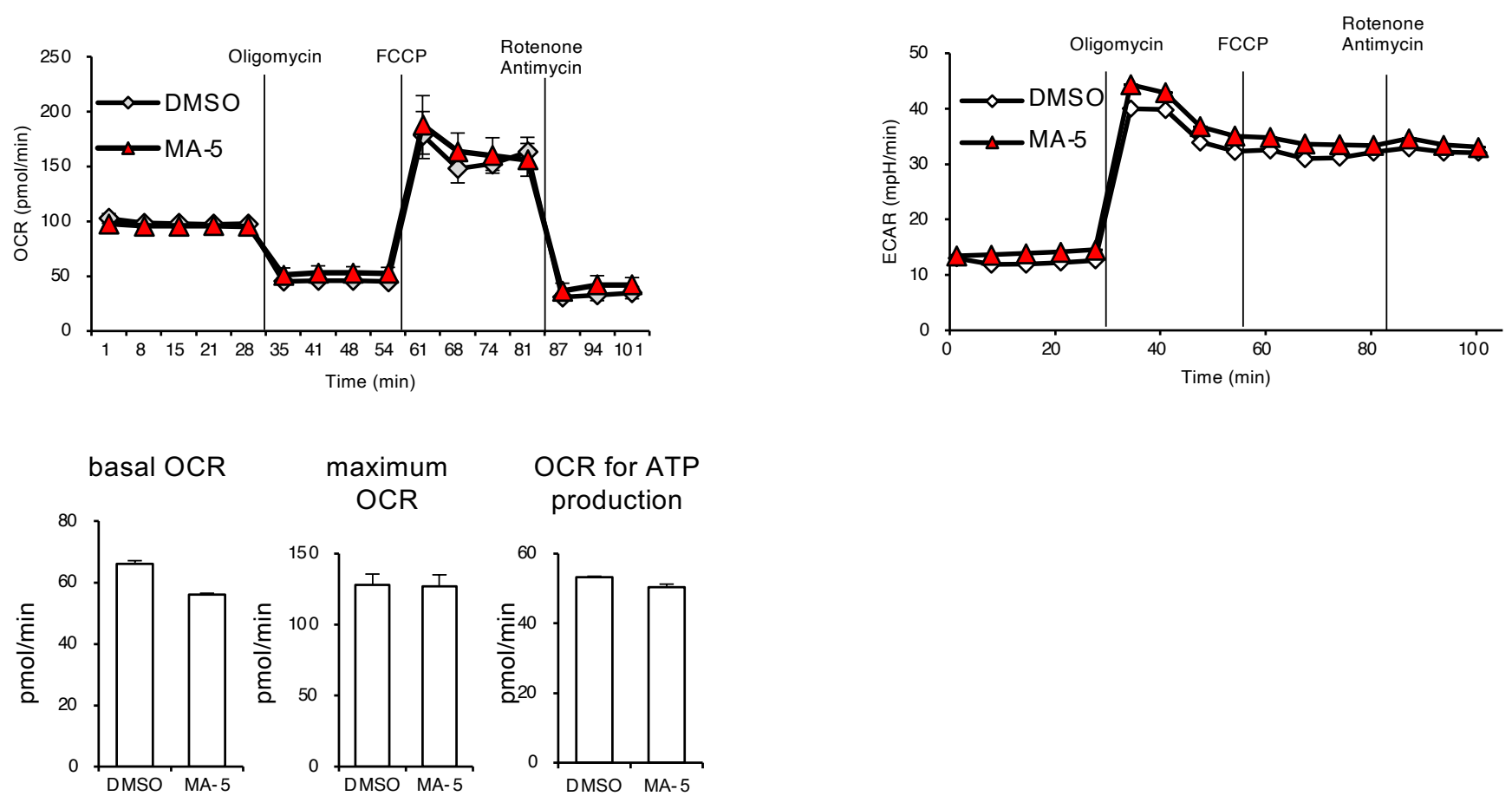

\section{Figure S3}

Effect of MA-5 on mitochondrial respiration.

Bioenergetic assay with myoblasts from sIBM patient (case2, case3) treatment DMSO and MA-5 in comparison with OCR (left) and ECAR (right). The data represent the mean $\pm \mathrm{SD}$. 


\section{Supplementary Figure 4}

\section{Case 2}
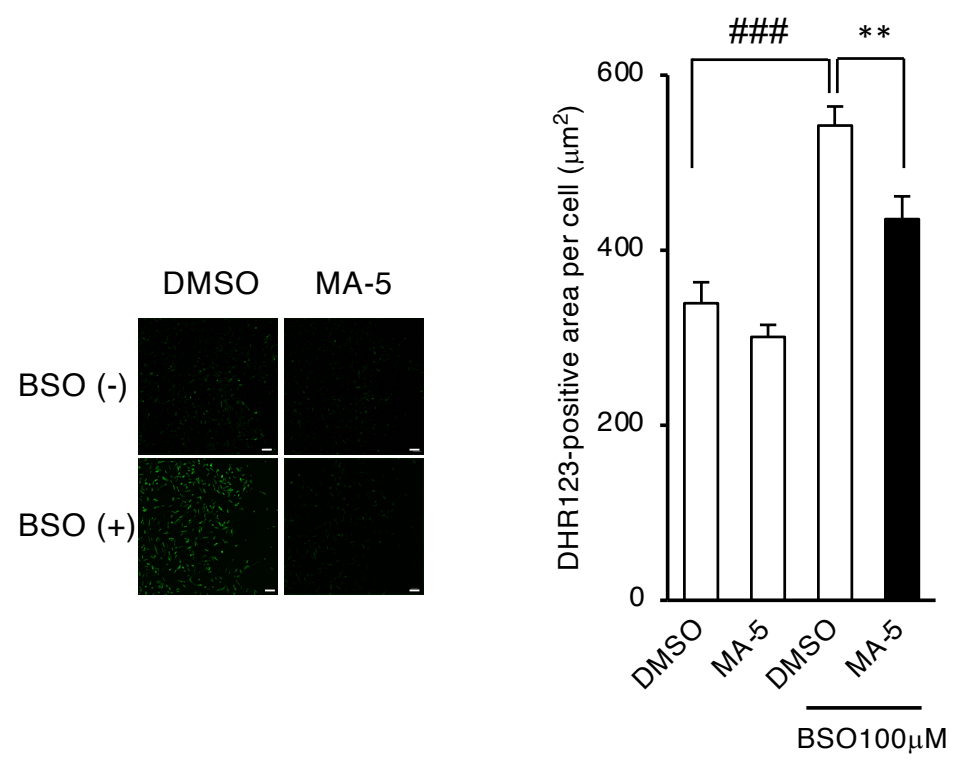

\section{Case 3}
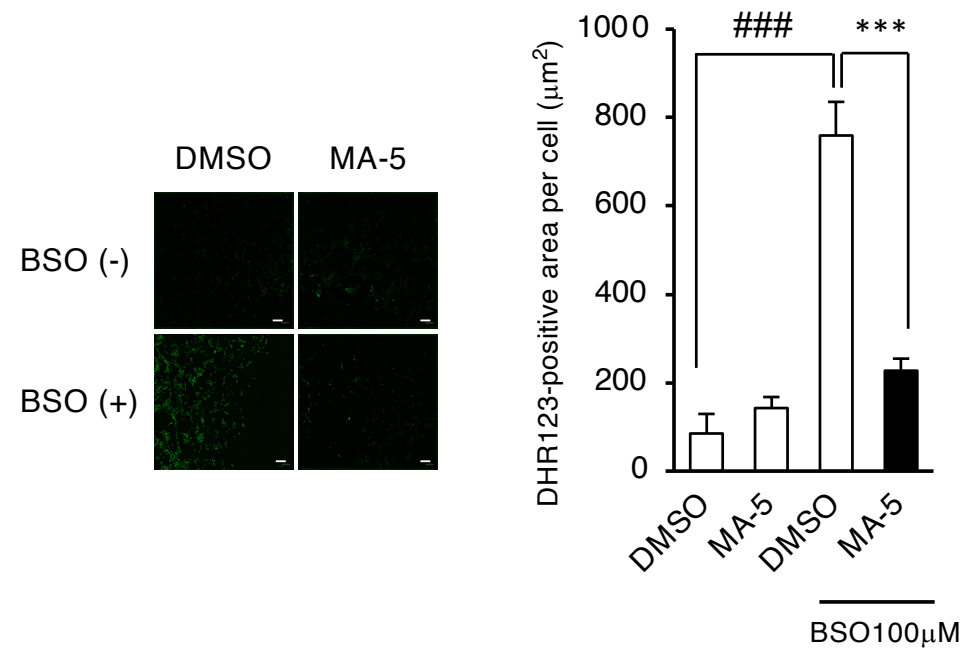

\section{Figure S4}

Effect of MA-5 on mtROS.

MA-5 improved ROS production by BSO. DHR123 staining of sIBM myoblasts with DMSO (upper left), MA-5 (upper right), BSO + DMSO (lower left), BSO + MA-5 (lower right). The data represent the mean \pm SEM.\#\#\#p $<0.001$ (unpaired twotailed Student's $t$-test versus DMSO), ${ }^{* *} p<0.01,{ }^{* * *} p<0.001$ (unpaired two-tailed Student's $t$-test versus BSO + DMSO). Scale bars represent $200 \mu \mathrm{m}$. 


\section{Supplementary Figure 5}

\section{A. $0.1 \%$ DMSO}
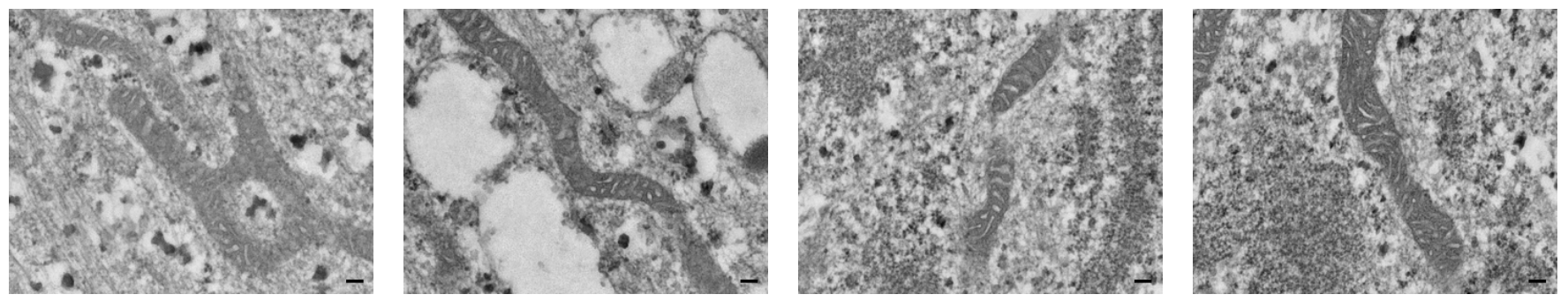

\section{B. MA-5 $10 \mu \mathrm{M}$}
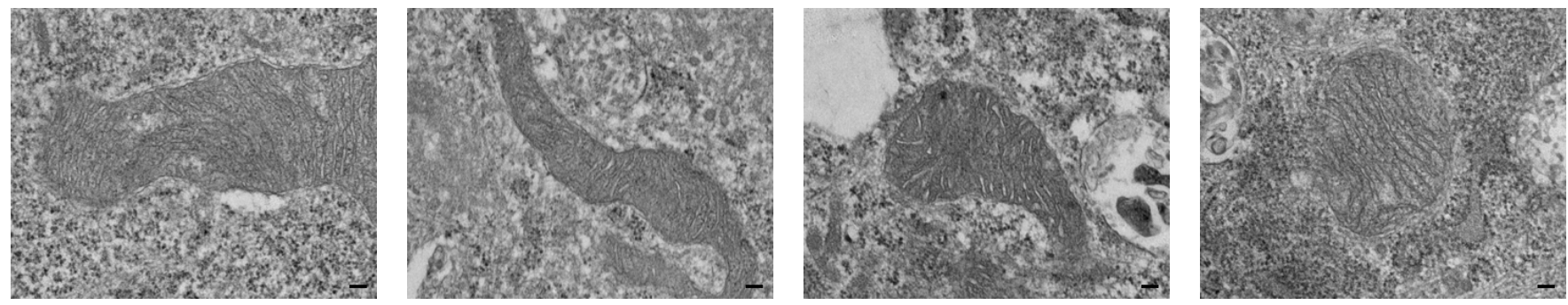

\section{Figure S5}

Electron microscopy of sIBM myoblasts.

Structural analysis of cristae of sIBM myoblasts by electroscopic analysis.

The sIBM myoblasts were treated with (A) $0.1 \%$ DMSO (B) $10 \mu \mathrm{M}$ MA-5 for $24 \mathrm{~h}$.

The comparison with maximal width and length of cristae measured by ImageJ software $(n=100)$. Scale bars represent $500 \mathrm{~nm}$ 


\section{Supplementary Figure 6}

A

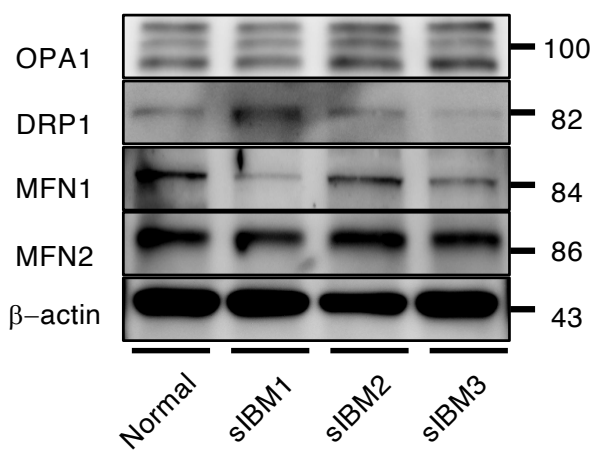

B. sIBM1 myoblast

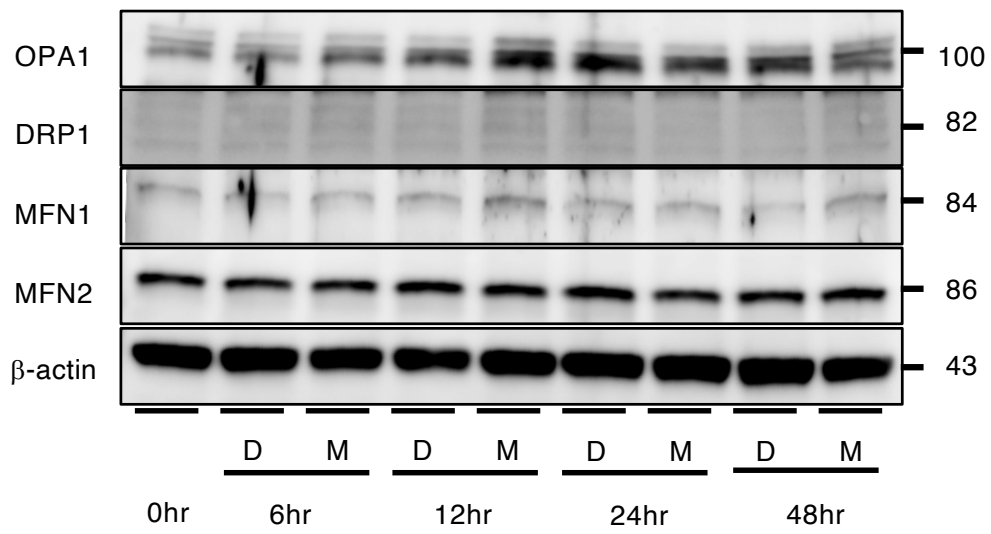

\section{C. sIBM2 myoblast}

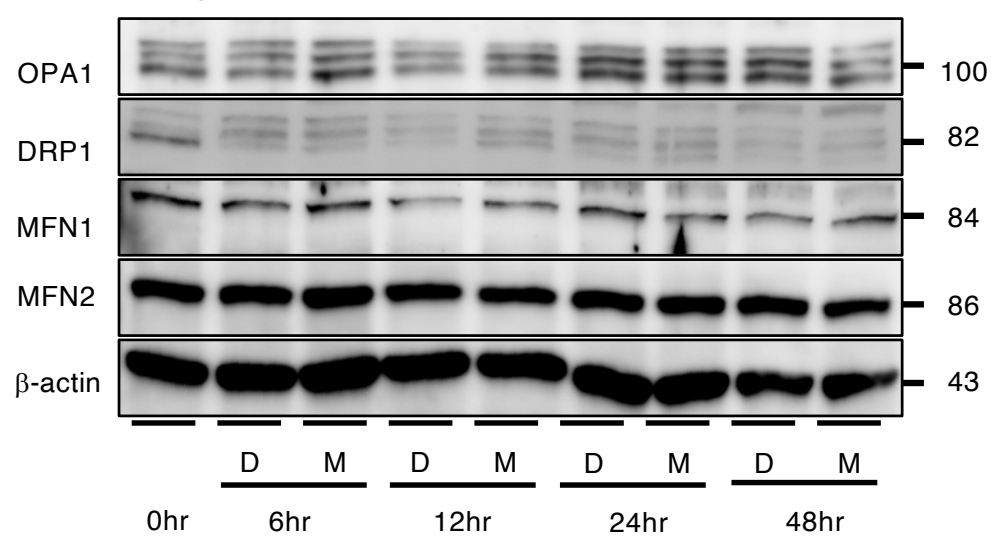

\section{D. sIBM3 myoblast}

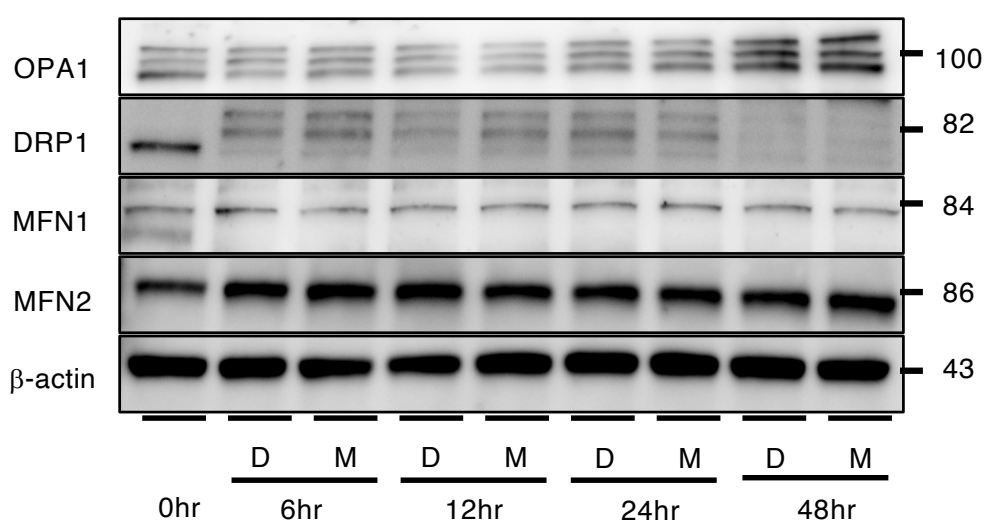

\section{Figure S6}

Effect of MA-5 on Mitochondrial related protein

A. Western blotting of individual myoblasts from normal control and sIBM patients for OPA1, DRP1, MFN1, MFN2. $\beta$-actin was used as a loading control.

B. Protein expression change in sIBM1

C. Protein expression change in sIBM2.

D. Protein expression change in sIBM3 


\section{Supplementary Figure 7}

\section{A. sIBM1 myoblast}

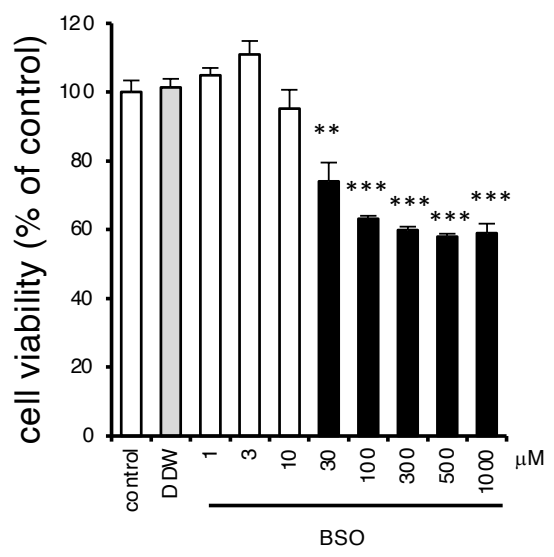

B. sIBM2 myoblast

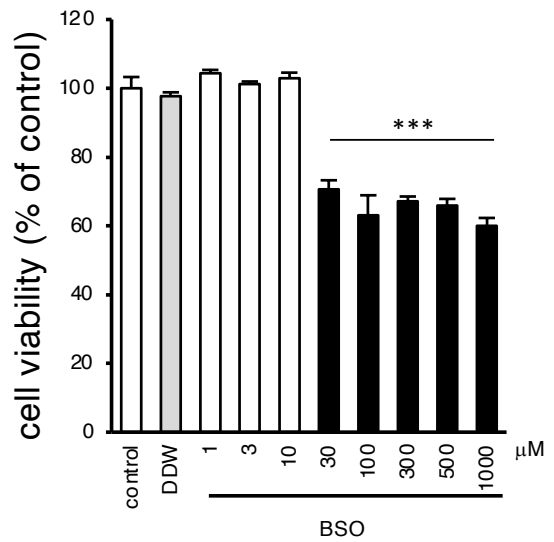

\section{C. sIBM3 myoblast}

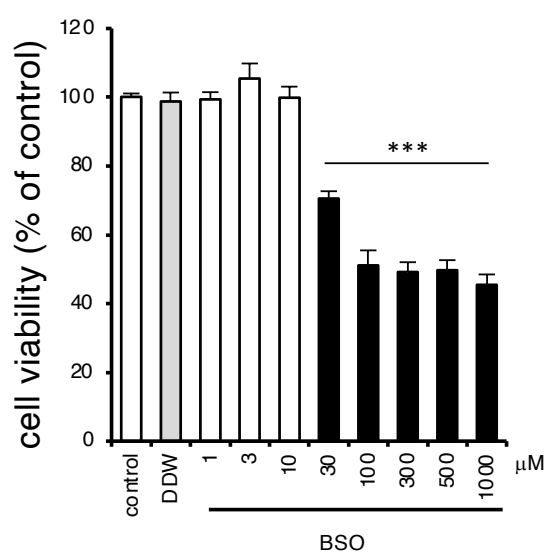

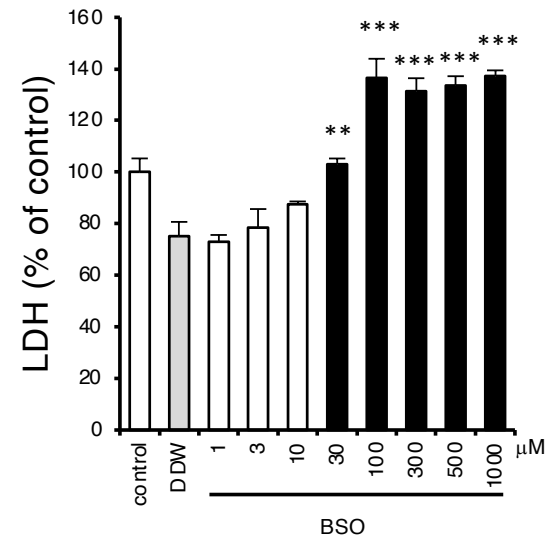
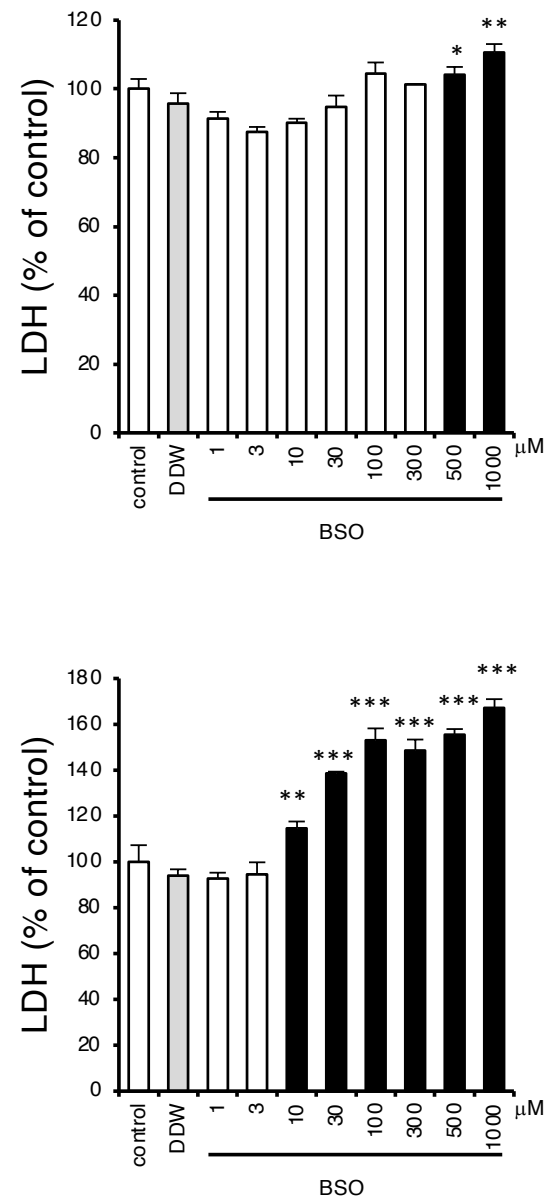

\section{Figure S7}

Cell death by BSO-treatment.

The figure on the left is the cell viability assay and on the right is the level of LDH in culture medium under oxidative stress induced by BSO-treatment. The data represent the mean \pm SEM. ${ }^{*} p<0.05$, $* * p<0.01$ and $* * * p<0.001$ (unpaired two-tailed Student's $t$-test versus DDW, $\mathrm{n}=4$ ). The black square indicates significantly increased or decreased compared with DDW. 
A. Normal myoblast
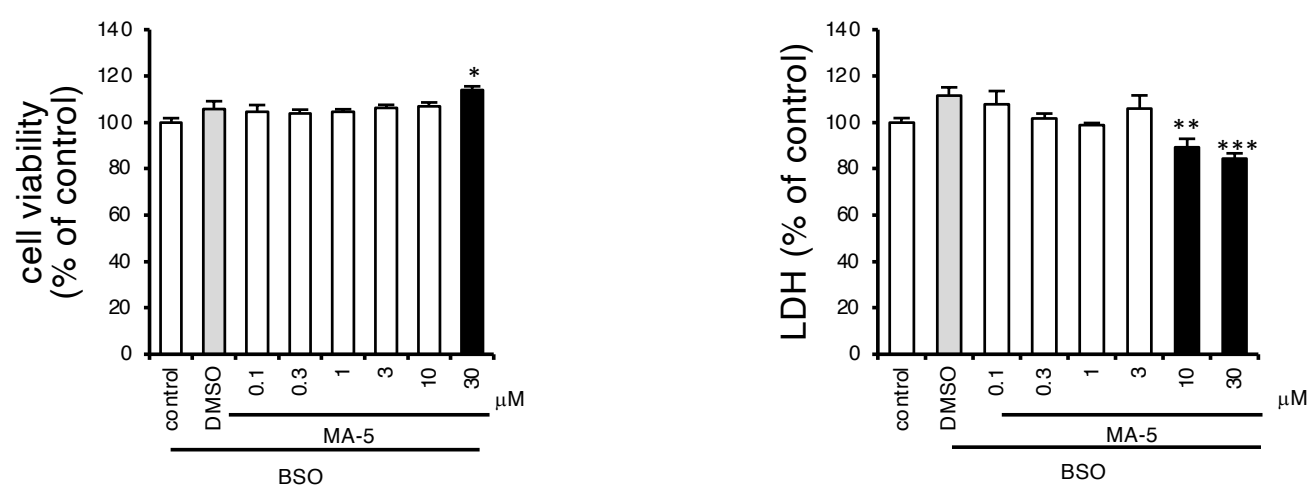

B. sIBM1 myoblast
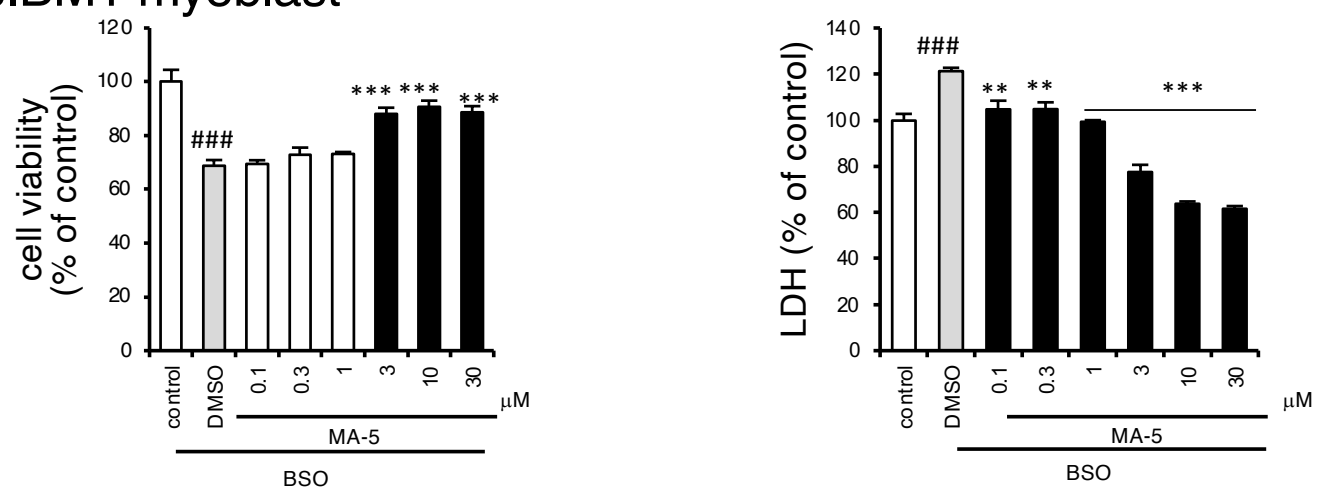

C. sIBM2 myoblast
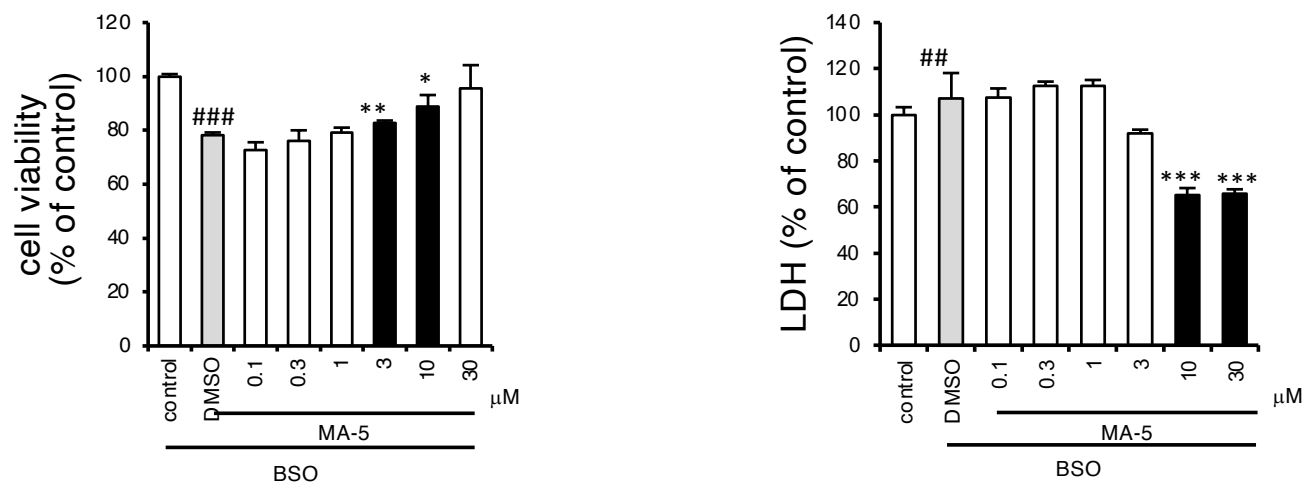

D. sIBM3 myoblast
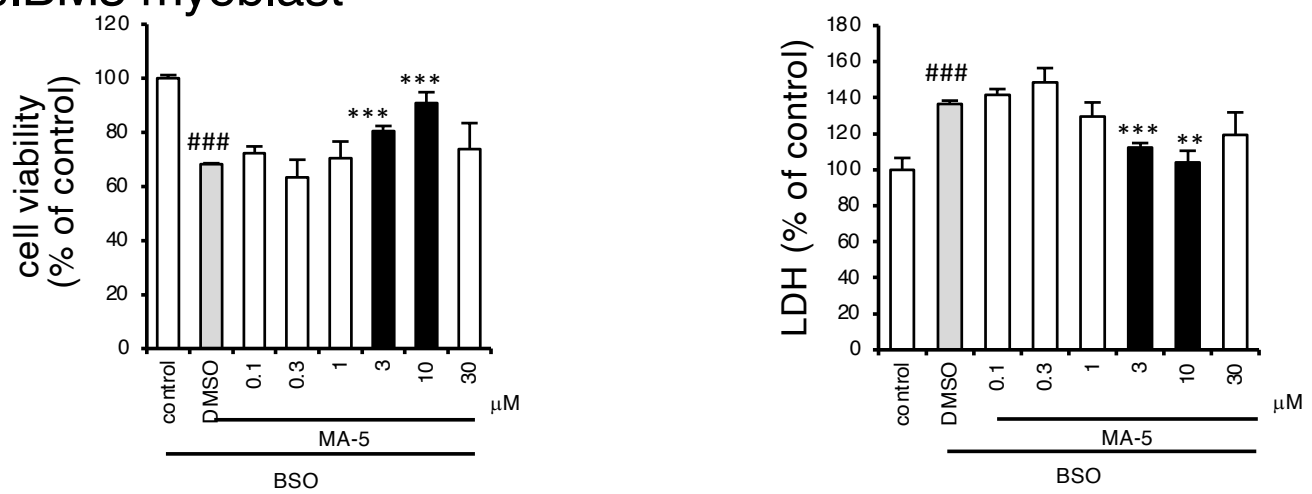

Figure S8

Cell-protective of MA-5 in sIBM myoblasts. The figure on the left is the cell viability assay and on the right is the level of LDH in culture medium under oxidative stress induced by BSO-treatment. The BSO concentration required to reach approximately $50-80 \%$ cell death varied with the myoblasts type. The data represent the mean \pm SEM. \#\#p $<0.01$ and \#\#\#p $<0.001$ (unpaired two-tailed Student's $t$-test versus control; $\mathrm{n}=4) .{ }^{*} p<0.05, * * p<0.01$ and $* * * p<0.001$ (unpaired two-tailed Student's $t$-test versus BSO+DMSO, $\mathrm{n}=4$ ). The black square indicates significantly increased or decreased compared with DMSO. 


\section{Supplementary Figure 9}

\section{A. $0.1 \%$ DMSO}
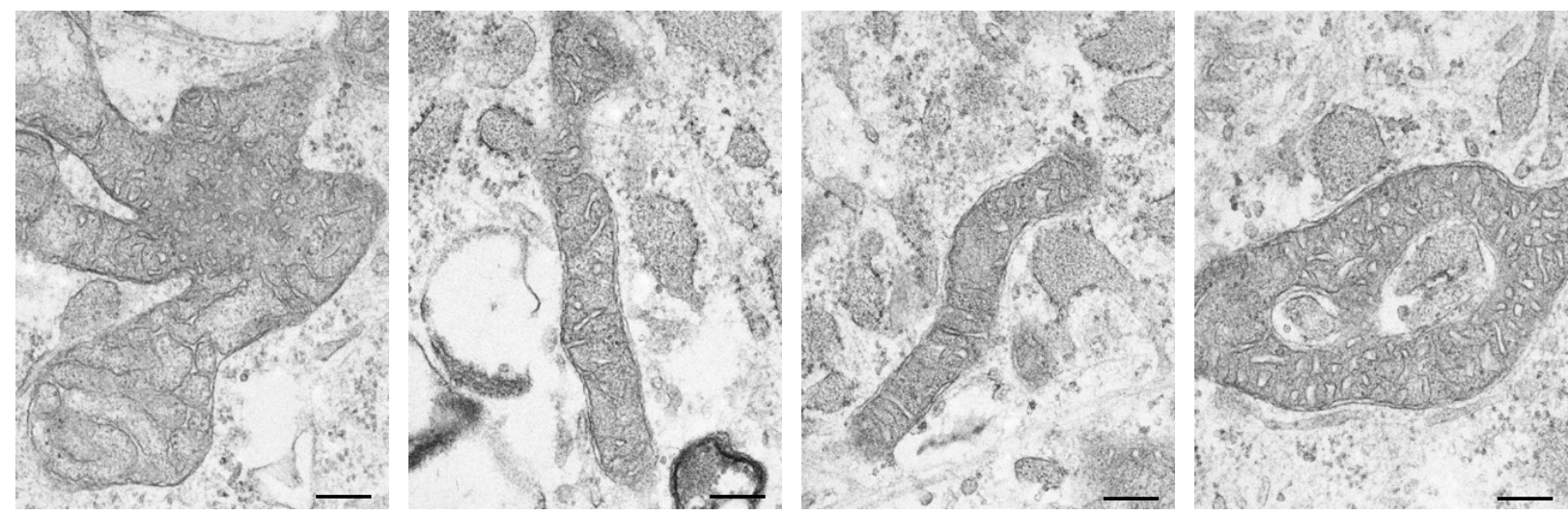

\section{B. MA-5 $10 \mu \mathrm{M}$}
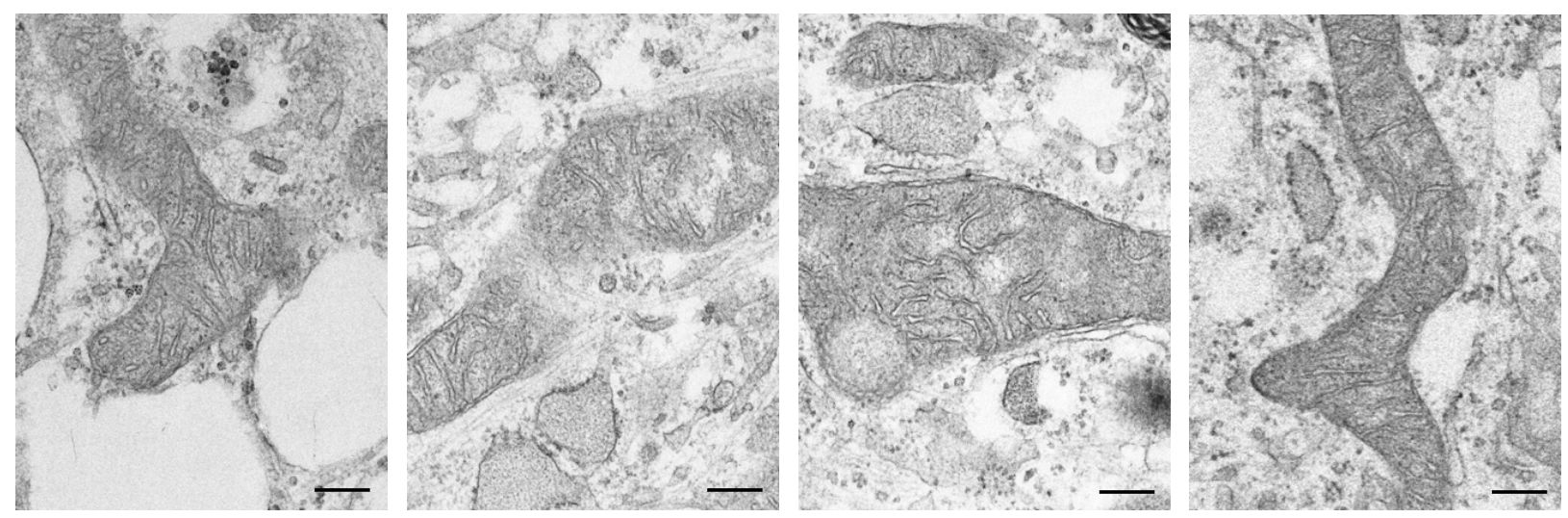

\section{Figure S9}

Electron microscopy of sIBM fibroblasts.

Structural analysis of cristae of sIBM fibroblasts by electroscopic analysis.

The sIBM fibroblasts were treated with (a) $0.1 \%$ DMSO (b) $10 \mu \mathrm{M}$ MA-5 for $24 \mathrm{~h}$.

The comparison with maximal width and length of cristae measured by ImageJ software $(\mathrm{n}=100)$. Scale bars represent $200 \mathrm{~nm}$ 


\section{Table S1}

\begin{tabular}{cccc}
\hline case & cell ID & pathogenic & non pathogenic \\
\hline 1 & sIBM1 & - & m.1438A $>\mathrm{G}$ \\
& & & m.1382A $>\mathrm{C}$ \\
2 & sIBM2 & - & $\begin{array}{l}\text { m.1438A }>\mathrm{G} \\
\text { 3.3849G }>A\end{array}$ \\
& & & m.1438A $>\mathrm{G}$ \\
\hline
\end{tabular}




\section{Table S2}

\section{A. cDNA taqman primer}

\begin{tabular}{cc}
\hline Gene (Human) & \\
\hline OPA1 & Hs01047018_m1 \\
MFN2 & Hs00208382_m1 \\
GAPDH & Hs02758991_g1 \\
\hline
\end{tabular}

\section{B. cDNA SYBR green primer}

\begin{tabular}{cc}
\hline Primer (Human) & Source \\
\hline DRP1 Forward: TCAACCTCCGTCTACTC & Nihon Gene Research Laboratories \\
DRP1 Reverse: GATCTGGAACTCGATGTCGGG & Nihon Gene Research Laboratories \\
\hline
\end{tabular}




\section{Table S3}

A. PCR primers for mitochondrial analysis by NGS

\begin{tabular}{lc}
\hline MTL-F1 & 5'-AAAGCACATACCAAGGCCAC-3' \\
MTL-F2 & 5'-TATCCGCCATCCCATACATT-3' \\
MTL-R1 & 5'-TTGGCTCTCCTTGCAAAGTT-3' \\
MTL-R2 & 5'-AATGTTGAGCCGTAGATGCC-3' \\
\hline
\end{tabular}

